\title{
Dynamics of genetic rescue in inbred Drosophila melanogaster populations
}

\author{
R. Bijlsma $\cdot$ M. D. D. Westerhof $\cdot$ L. P. Roekx • \\ I. Pen
}

Received: 11 October 2009/Accepted: 23 December 2009/Published online: 4 February 2010

(C) The Author(s) 2010. This article is published with open access at Springerlink.com

\begin{abstract}
Genetic rescue has been proposed as a management strategy to improve the fitness of genetically eroded populations by alleviating inbreeding depression. We studied the dynamics of genetic rescue in inbred populations of Drosophila. Using balancer chromosomes, we show that the force of heterosis that accompanies genetic rescue is large and allows even a recessive lethal to increase substantially in frequency in the rescued populations, particularly at stress temperatures. This indicates that deleterious alleles present in the immigrants can increase significantly in frequency in the recipient population when they are in linkage disequilibrium with genes responsible for the heterosis. In a second experiment we rescued eight inbred Drosophila populations with immigrants from two other inbred populations and observe: (i) there is a significant increase in viability both 5 and 10 generations after the rescue event, showing that the increase in fitness is not transient but persists long-term. (ii) The lower the fitness of the recipient population the larger the fitness increase. (iii) The increase in fitness depends significantly on the origin of the rescuers. The immigrants used were fixed for a conditional lethal that was mildly
\end{abstract}

R. Bijlsma (ه) · M. D. D. Westerhof · L. P. Roekx

Population and Conservation Genetics, Centre for Ecological and Evolutionary Studies, University of Groningen, P.O. Box 14, 9750 AA Haren, The Netherlands

e-mail: r.bijlsma@rug.nl

R. Bijlsma · I. Pen

Theoretical Biology, Centre for Ecological and Evolutionary Studies, University of Groningen, P.O. Box 14, 9750 AA Haren, The Netherlands

R. Bijlsma

Department of Biological Sciences, Ecology and Genetics, Aarhus University, Ny Munkegade, Buildg. 1540, 8000 Aarhus C, Denmark deleterious at $25^{\circ} \mathrm{C}$ but lethal at $29^{\circ} \mathrm{C}$. By comparing fitness at $25^{\circ} \mathrm{C}$ (the temperature during the rescue experiment) and $29^{\circ} \mathrm{C}$, we show that the lethal allele reached significant frequencies in most rescued populations, which upon renewed inbreeding became fixed in part of the inbred lines. In conclusion, in addition to the fitness increase genetic rescue can easily result in a substantial increase in the frequency of mildly deleterious alleles carried by the immigrants. This can endanger the rescued population greatly when it undergoes recurrent inbreeding. However, using a sufficient number of immigrants and to accompany the rescue event with the right demographic measures will overcome this problem. As such, genetic rescue still is a viable option to manage genetically eroded populations.

Keywords Drosophila - Gene flow - Genetic drift . Genetic load · Genetic rescue - Inbreeding .

Inbreeding depression

\section{Introduction}

Human impact on nature has caused habitats to become increasingly fragmented. As a result, populations of many species have become small and greatly isolated (Ceballos and Ehrlich 2002). Typically, such populations are subject to genetic drift and inbreeding with associated decline in fitness due to inbreeding depression (Frankham 1995; Hedrick and Kalinowski 2000). This fitness decrease is for the greater part thought to be caused by fixation of recessive detrimental variation (Hedrick 1994; Charlesworth and Charlesworth 1999; Wang et al. 1999) thereby significantly elevating the extinction risk of populations (Newman and Pilson 1997; Saccheri et al. 1998; Bijlsma et al. 2000). Although the importance of inbreeding depression in 
conservation biology has been hotly debated (Caughley 1994; Hedrick et al. 1996), there is now increasing evidence that inbreeding depression is present in population of many wild living species (Keller and Waller 2002) and does significantly contribute to the extinction risk of populations (Hedrick 1995; Spielman et al. 2004; Liberg et al. 2005).

To prevent populations to become extinct from inbreeding, inbred populations may be genetically rescued and have their fitness partly restored by immigration of a few unrelated individuals from another population (Tallmon et al. 2004; Hedrick 2005; Edmands 2007). This is for the greater part due to the fact that the infusion of unrelated genomes increases heterozygosity in the recipient population, thereby reducing the inbreeding load caused by the fixation of recessive deleterious alleles, though other processes can play a significant role (e.g. frequency dependent selection for rare $S$-alleles in plants (Leducq et al. 2010)). This concept of genetic rescue is now considered to be a realistic management option to counteract the increased extinction risk from genetic erosion (for reviews see Tallmon et al. 2004; Hedrick 2005; Edmands 2007), and has been shown to be very effective in a number of cases (Hedrick 1995; Westemeier et al. 1998; Madsen et al. 1999; Willi et al. 2007; Bouzat et al. 2009; Hedrick and Fredrickson 2010).

However, this process is not without potential dangers (see Tallmon et al. 2004; Edmands 2007; Hedrick and Fredrickson 2010). First, immigration by genetically divergent individuals can disrupt both intrinsic coadaptation and local adaptation (Hedrick 1995; Edmands 2007) and result in outbreeding depression (Dobzhansky and Pavlovsky 1958). To what extent these potential fitness costs of genetic rescue are larger than the fitness benefits is, therefore, an important issue in conservation genetics. Second, when the immigrants are successful their genomes can increase rapidly and disproportional in frequency in the recipient population in a few generations (Ball et al. 2000; Ebert et al. 2002; Saccheri and Brakefield 2002). As such, the immigrant genomes become overrepresented in the rescued population thereby reducing the genetically effective population size, $N_{e}$, rather than increasing it (Hedrick and Fredrickson 2010). Third, the fitness benefits may be short-lived as recombination may dilute the heterotic effect in subsequent generations (Lynch 1991).

The aim of this paper is to explore some of the potential dangers of genetic rescue using Drosophila melanogaster as a model organism. Based on the assumption that heterosis from covering up the expressed load from deleterious mutations in the rescued population leads to a rapid spread of the immigrant genomes (Ball et al. 2000; Saccheri and Brakefield 2002), we hypothesize that this not only holds for the loci that are under positive selection but also for genes in linkage disequilibrium with these loci (Maynard Smith and Haigh 1974). Moreover, if the heterotic selection pressure is strong enough even rare deleterious alleles present in the immigrants and linked to the genes that are responsible for the heterosis may reach appreciable frequencies in the rescued populations. In later generations this may cause additional fitness problems, particularly when $N_{e}$ is not greatly increased by the rescue event. In the first experiment we explore this possibility by "rescuing" ten independently inbred populations of D. melanogaster by immigrants that carry chromosome balancers that are lethal in homozygous condition. We show that in some cases such chromosome balancers do significantly increase in frequency despite the fact that these chromosomes carry a recessive lethal.

To test if genetic rescue leads to a more long-term fitness increase, we rescue inbred populations with immigrants from either one of two other inbred populations that carry a conditional recessive lethal system that is not expressed at a benign temperature but causes high pre-adult mortality under high temperature stress. We assess the increase in viability (the rescue effect) compared to the non-rescued populations at two different time points, five and ten generations after the rescue event. We show that a positive rescue effect is still observed after ten generations but that the magnitude varies considerably between both the different rescued populations and the assay temperatures.

Applying genetic rescue as a management measure is primarily meant to increase the fitness of the rescued population and to increase the population numbers in the long-term. Therefore, genetic measures have necessarily to be complemented by demographic and environmental measures (Robert et al. 2007). However, given the current level of habitat fragmentation, the population size probably may increase to a certain extent, but still will be (very) limited. In these situations recurrent inbreeding cannot be prevented and the resulting genetic deterioration may become as bad or even worse than before. This has been observed in an isolated population of gray wolves in Scandinavia that was initially rescued by a single migrant where after the population numbers increased (Vilà et al. 2002). More recently, however, this population has been shown again to be severely inbred and its persistence is greatly endangered due to inbreeding depression (Liberg et al. 2005). To investigate the consequences of recurrent inbreeding we subject rescued populations to renewed inbreeding and test the fitness of the resulting inbred lines again at 25 and $29^{\circ} \mathrm{C}$. We observe that the introgressed conditional lethal can become prevailing in this situation.

\section{Materials and methods}

Flies and inbreeding procedure

The inbred flies used for the experiments originated from the Groningen' 83 base population. This population was 
founded by 403 inseminated females caught at a local fruit market in Groningen (The Netherlands) in 1983 and kept as a large population since then (see Vermeulen and Bijlsma 2006). Previous experiments have shown that this population still exhibits substantial levels of genetic variation and still harbours a considerable load (Bijlsma et al. 1999; Vermeulen and Bijlsma 2004a, b). Flies were normally maintained on standard medium $(1,000 \mathrm{ml}$ water, $32 \mathrm{~g}$ dead yeast, $54 \mathrm{~g}$ sucrose, $18 \mathrm{~g}$ agar and $13 \mathrm{ml}$ nipagin solution (10 g nipagin in $100 \mathrm{ml}$ ethanol)) at $25^{\circ} \mathrm{C}, 50 \%$ R.H. and constant light.

Inbred lines were established from the base population through four consecutive generations of full-sib mating resulting in a theoretically inbreeding level of $f=0.594$ (Falconer and Mackay 1996). To this end, single pairs of flies were randomly established in vials and from the offspring of each pair five full-sib pairs were established individually in vials. For the next round of inbreeding again five single pairs were established from that of the five vials of the previous generation that on visual inspection produced the most offspring. This procedure selects for high productivity and allows for within line purging of highly deleterious alleles (Wang 2000). This mimics in part the process of purging as it would occur in small populations and avoids that ending up with greatly crippled lines that would never occur under natural conditions. When reaching the desired level of inbreeding, the lines were expanded and maintained as bottle populations (300-400 individuals per generation) for several generations prior to the experiments. Of these lines the first ten remaining (I(nbred)1, I3, I5, I6, I7, I8, I11, I12, I15 and I17) were used for the experiments described here. Throughout the paper, we will refer to these as inbred populations.

\section{Balancer chromosome introgression experiment}

To test whether or not deleterious alleles can invade in an inbred population we used the 2 nd chromosome balancer $\mathrm{CyO}$ and the 3rd chromosome balancer TM3. Chromosomes 2 and 3 are marked with visible (dominant) mutations curled wings (Cy) and serrate wings (Ser), respectively (for description of balancers and markers see Lindsley and Zimm 1992). The balancer chromosomes contain a series of overlapping inversions that prevent successful recombination along the chromosome and consequently the chromosome is inherited to next generation as a unit. In addition, both balancers carry a recessive embryonic lethal, meaning that homozygotes for each of the balancers do not develop.

By the crossing procedure outlined in Fig. 1 we produced females that were heterozygous for both balancers in an otherwise inbred genetic background (except for the

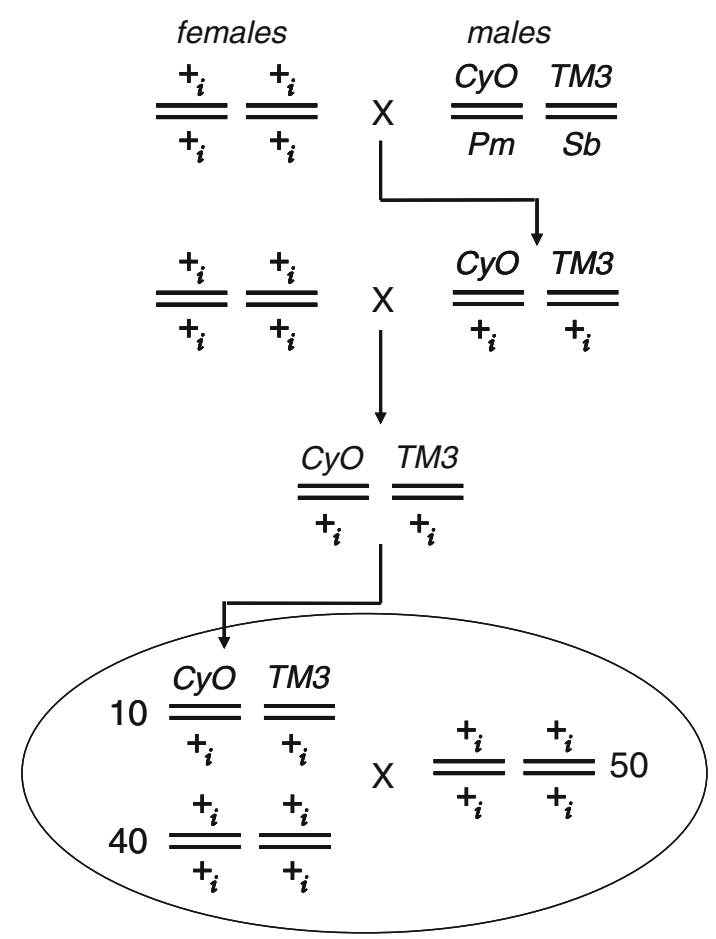

Fig. 1 Crossing scheme to produce females that are heterozygous for the balancer chromosomes $\mathrm{CyO}$ and TM3 in an inbred background (top).$+{ }_{i}$ Denotes chromosomes originating from the inbred population $i$. Note that this scheme ensures that the $\mathrm{X}$-chromosomes are all originating from the inbred population. The bottom part (within the oval) shows the initial composition of the rescued populations

very small 4th chromosome) for each of the inbred populations separately. The X-chromosomes all originate from the inbred population. These females were used as migrants and for each inbred population 10 of these females, virgins, were combined with 40 virgin females and 50 males from the inbred population and used as initial parents for the rescued populations (Fig. 1). This results in an initial frequency of each of the balancers of $5 \%$ in each population. The next generation, all offspring were transferred to a new bottle to serve as parents for the next generation. After a sufficient number of eggs was produced (200-500 eggs per bottle) these flies were removed and phenotypically scored for the presence (heterozygotes) or absence (wildtype inbred homozygotes) of the balancers. For each of the ten different inbred populations two sets of three replicate bottles were established and one set was placed at $25^{\circ} \mathrm{C}$ and the other at $29^{\circ} \mathrm{C}$. In the first generation we observed problems with maintaining the populations at $29^{\circ} \mathrm{C}$ probably due to a high incidence of male sterility that can occur at this temperature (Rohmer et al. 2004). Therefore, the offspring of the bottles at $25^{\circ} \mathrm{C}$ were transferred twice to new bottles for egg laying and one replicate was placed at a somewhat lower stress temperature of $28.5^{\circ} \mathrm{C}$. 
Prior to the experiment, the ten inbred populations, and two additional inbred populations that were in the end not used for the experiments described here, were intercrossed for at least ten generations to produce a large outbred MIX population that served as a control population. For this population, ten replicate bottle populations were initiated with a frequency of $5 \%$ of both balancers at the start of the experiment and maintained in a similar way as the inbred populations. For all populations and at both temperatures, the balancer frequencies were determined for eight generations.

The rationale behind this experiment is outlined by Sved and Ayala (1970) and has been successfully applied by Bijlsma et al. (1999). In short, balancer homozygotes are lethal and the wildtype homozygotes may also have a decreased fitness because of inbreeding depression. The latter, therefore could be at a disadvantage compared to the balancer heterozygotes. If the overall fitness of the balancer is greater than that of both homozygotes (the classical overdominance model) then the balancer heterozygote can introgress in the rescued population and will reach a stable equilibrium in the population. The frequency ultimately reached, depends on the degree of inbreeding depression displayed by the inbred homozygotes.

\section{Rescue experiment}

In a separate experiment we studied the effect of immigration on the viability of the original inbred populations using females of the inbred populations I12 and I15 as immigrants to "rescue" the remaining eight inbred populations (as such $\mathrm{I} 12$ and I15 from then on are denoted as R12 and R15, respectively). These two populations were chosen as rescuers as they were found to be fixed for a recessive conditional lethal system that causes almost $100 \%$ mortality in the pupal stage at $29^{\circ} \mathrm{C}$ but has no or little effect on pupal survival at $25^{\circ} \mathrm{C}$ (see "Results" section for details). Such conditional lethals seem to be present in many Drosophila species and populations (Dobzhansky et al. 1955; Tobari 1966; Vermeulen and Bijlsma 2004a, b; Bakker et al. 2010) and have been observed before in low frequencies in the Groningen' 83 base population (unpublished data). From this we infer that these conditional lethals are mildly deleterious at $25^{\circ} \mathrm{C}$ and maintained in a mutation-selection balance in this base population. As the rescue experiment and the concurrent renewed inbreeding experiment are done at $25^{\circ} \mathrm{C}$, we can follow the increase of the frequency of the conditional lethals by testing pre-adult or pupal survival at both 25 and $29^{\circ} \mathrm{C}$ : an increase in the frequency of the lethal will result in an increase in pre-adult mortality at $29^{\circ} \mathrm{C}$, even though a small part of this increase might be attributed to the increase in inbreeding level (see "Results").
The rescue experiment is outlined in Fig. 2. It was initiated by combining ten virgin females of either R12 or R15 with 40 virgin females and 50 males of each of the remaining 8 inbred populations to start bottle populations with an initial frequency of $10 \%$ of the rescue genome, the so called rescued populations. For each combination of rescuer and inbred population three replicates were established at $25^{\circ} \mathrm{C}$. From these bottles, the offspring were transferred to new bottles as parents for the next generation every fortnight. The original populations, further denoted as non-rescued populations, were maintained as single populations at the same time (for each population five bottles that were mixed every generation).

To assess the effect of immigration, viability (egg-toadult survival) was determined for the rescued and nonrescued populations five and ten generations after the rescue event (Fig. 2). From each replicate population five vials with 50 eggs each were established and viability was calculated as the fraction of the eggs that resulted in adult flies. For the non-rescued populations ten vials with 50 eggs each were initiated to determine viability. For each replicate rescued population, the rescue effect was calculated as being the average viability of the rescued replicate population minus the average viability of its non-rescued counter part (Rescue effect $\left.=V_{\text {Res }}-V_{\text {Non-R }}\right)$. Note, that we had a single viability estimate for each non-rescued population that was used for all three replicates of the corresponding rescued populations. The rescue effect was determined for the populations both at 25 and at $29^{\circ} \mathrm{C}$.

To determine to what extent the rescue event had affected the resistance to the high temperature stress, we additionally calculated the cost of stress. This is defined as the average viability of a population at $25^{\circ} \mathrm{C}$ compared to its average viability at $29^{\circ} \mathrm{C}\left(\right.$ Cost of stress $\left.=\mathrm{V}_{25^{\circ} \mathrm{C}}-\mathrm{V}_{29^{\circ} \mathrm{C}}\right)$.

Pupal mortality after renewed inbreeding

After generation ten, the consequence of renewed inbreeding on pupal mortality was assayed for all rescued and non-rescued populations. To this end for each population (both rescued and non-rescued) twenty independent pairs of flies were initiated and their offspring were full-sib mated for six additional generations, this time without replication (Fig. 2). One has to realize that for the nonrescued populations these six generations of inbreeding came on top of the four generations they already had undergone previously. During this process many of the full-sib lines became extinct before they reached the desired level of inbreeding (for population I5 none made it till the last generation). Consequently, the number of replicate full-sib lines varied greatly among the populations. After the inbreeding process was finished, of each inbred 


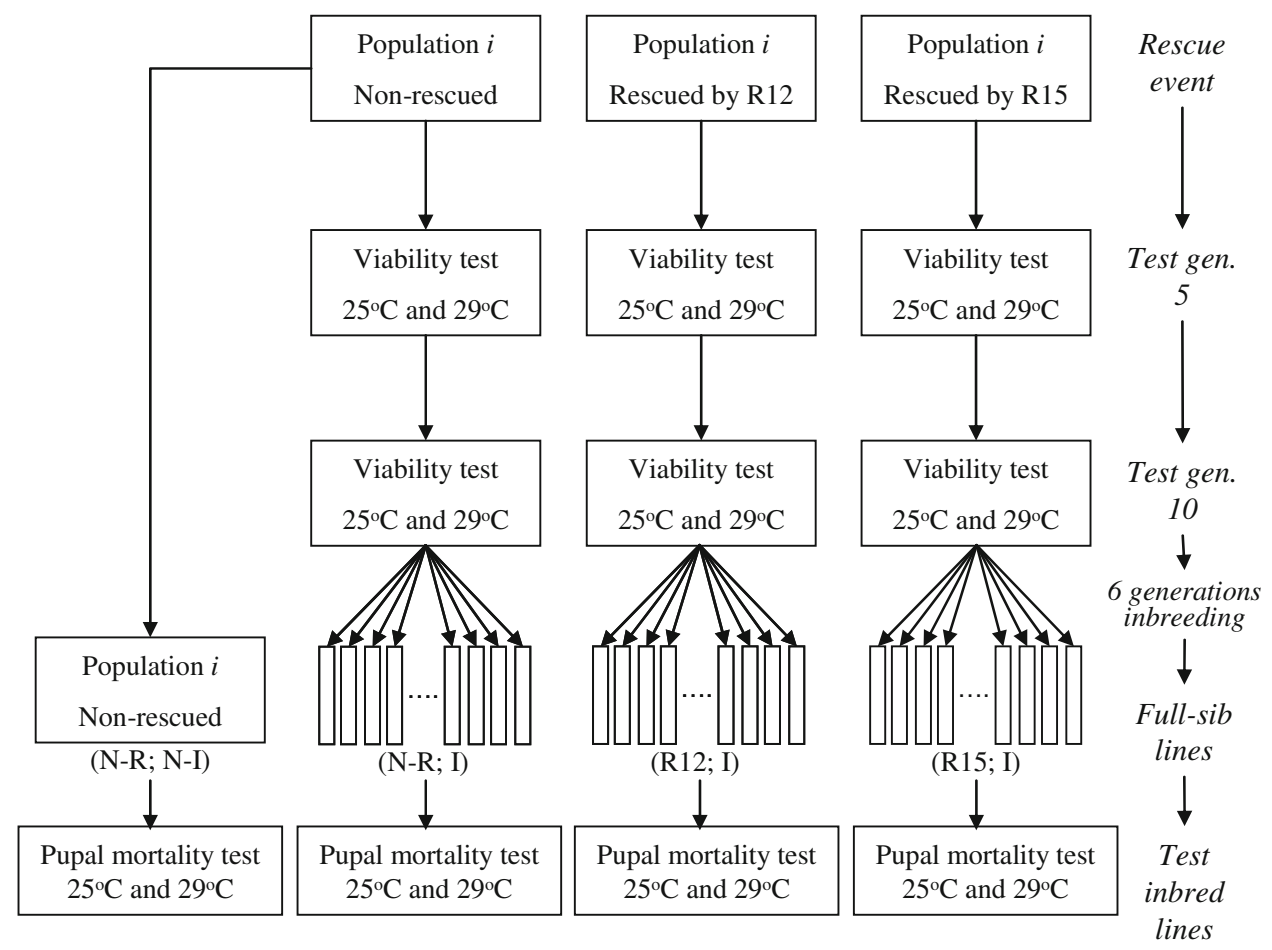

Fig. 2 Flow diagram of the rescue experiment and the concurrent renewed inbreeding. The different time points are: Rescue event gives the parental generation in which immigrant females were combined with each of eight inbred populations. Test generation 5: the rescue effect was determined five generations after the rescue event. Test generation 10: the rescue effect was determined ten generations after

line two vials were initiated with 100 eggs each, one vial was placed at $25^{\circ} \mathrm{C}$ and the other at $29^{\circ} \mathrm{C}$. For each vial the total number of pupae and the number of pupae that did not eclose was determined and pupal mortality was calculated as the fraction of pupae that did not eclose. The cost of pupal stress is defined as the pupal mortality at $29^{\circ} \mathrm{C}$ minus the mortality at $25^{\circ} \mathrm{C}$. For each rescued and non-rescued population the average over all full-sib lines was calculated. At the same time the ten original inbred populations that had not undergone any further manipulation were also assayed for pupal mortality at both temperatures (ten replicates with 100 eggs per vial for each population) and the cost of pupal stress was calculated for these populations also (Fig. 2).

Data analysis

For the rescue and pupal mortality experiment, viabilities and mortality rates were modeled with linear mixed models, using the linear mixed effects (lme) procedure from the nlme package in $\mathrm{R}$ 2.9.0 ( $\mathrm{R}$ Development Core Team 2005). All effects were included as fixed effects, except "population" which was included as a random effect. the rescue event. Six generations inbreeding: from the rescued and non-rescued populations inbred lines were established after the tenth generation by 6 generations of full-sib mating. Test inbred lines: pupal mortality was determined of all extant inbred lines after the sixth generation of inbreeding. This was also done for the original inbred populations

Models were fitted using a maximum likelihood approach and significance of fixed effects was assessed by means of log-likelihood ratio $\left(\chi^{2}\right)$ tests (Crawley 2007). We started model building with full models including interactions of all orders, using backward elimination to arrive at final models. The normality assumption was checked by visual inspection of residuals of final models and by using QQ plots. No strong deviations from normality were observed. For the pupal mortality assay, we did not develop the full model as the design was unbalanced. Therefore, the effects of recurrent inbreeding, rescued versus non-rescued populations, and R12 versus R15 were analysed separately.

\section{Results}

\section{Balancer introgression experiment}

Figure 3 shows the change in frequency of the balancer chromosomes at the three different treatments. At $25^{\circ} \mathrm{C}$ (Fig. 3a-b) the balancers do increase in frequency in two of the ten inbred populations. For I1 the frequency of the $\mathrm{CyO}$ balancer increases to a value higher than 0.25 , which 
Fig. 3 Changes in frequency during eight generations of the 2nd chromosome balancer $\mathrm{CyO}$ (left) and 3rd chromosome balancer TM3 (right) at three temperatures $25^{\circ} \mathrm{C}(\mathbf{a}-\mathbf{b})$, $28.5^{\circ} \mathrm{C}(\mathbf{c}-\mathbf{d})$ and $29^{\circ} \mathrm{C}(\mathbf{e}-\mathbf{f})$ when introduced in ten different inbred populations. Specifically marked populations are: I1 (crosses), I5 (diamonds), I12 (open circles) and $\mathrm{I} 15$ (open squares). The other six populations are marked by dots. The outbred MIX population is marked by triangles. Note, the scaling of the $y$ axis in the top figures is different from that of the other figures
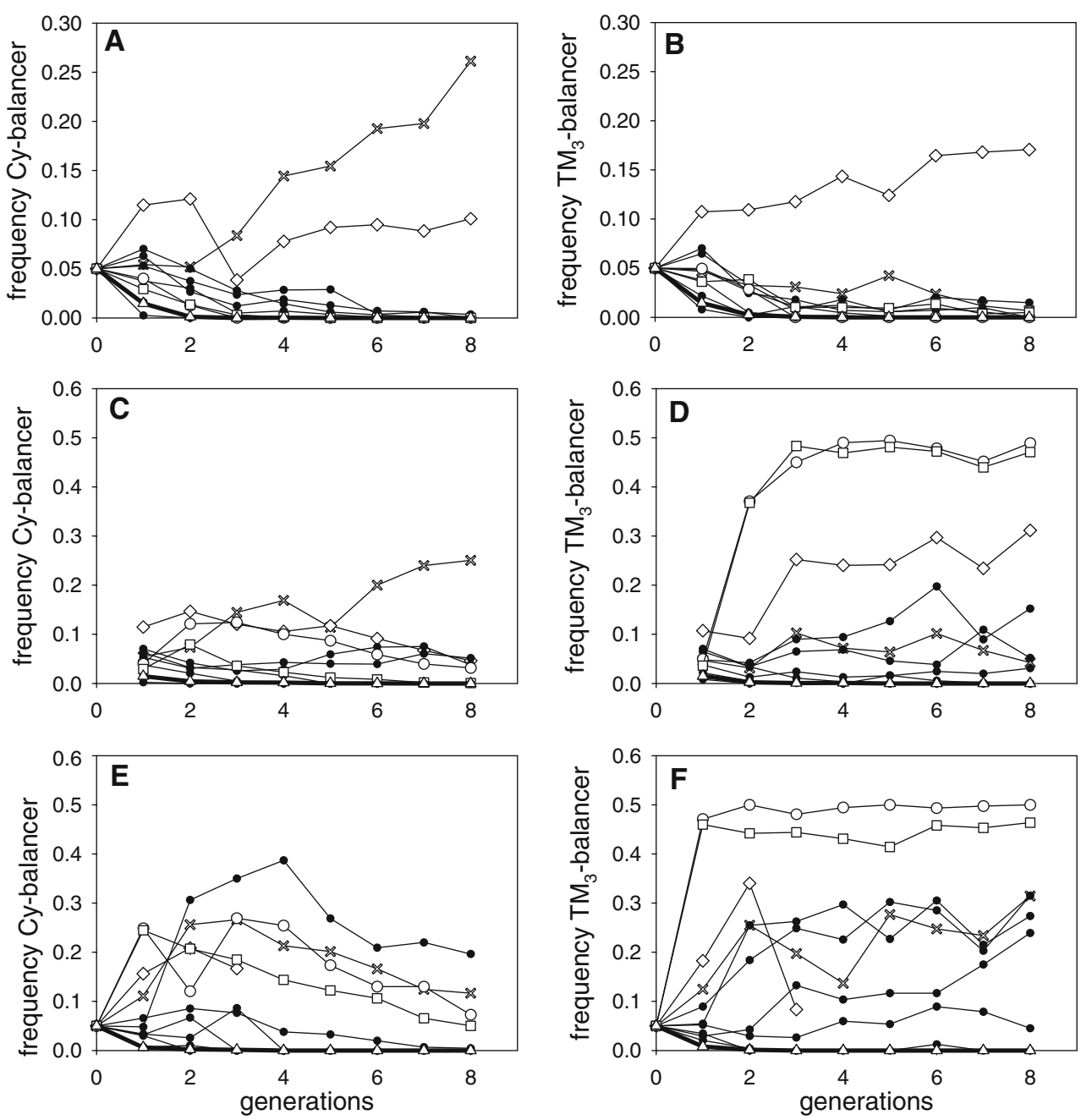

means that $50 \%$ of the flies are in fact heterozygous for this balancer. No increase for the TM3 balancer is observed in I1. Thus most inbreeding in this population can be attributed to deleterious genes at the 2nd chromosome. For I5 both balancers reach frequencies higher than the starting frequency, although the increase is less than observed for I1, indicating that in this inbred population both the 2 nd and the 3rd chromosome contribute to the inbreeding depression.

In contrast, the balancers are very rapidly lost in the outbred MIX population and in generation 3 the $\mathrm{CyO}$ balancer has disappeared from this population and TM3 is found only in one replicate population at a frequency of 0.0043 . For most other inbred populations the frequency of both balancers is also decreasing but at a clearly slower rate. Nevertheless, in two out of ten populations the force of heterosis is sufficiently large to facilitate introgression of even a recessive lethal.

When the temperature is increased to $28.5^{\circ} \mathrm{C}$ (Fig. 3c-d) or $29^{\circ} \mathrm{C}$ (Fig. 3e-f) the picture changes considerably. Still the balancers disappear rapidly from the outbred MIX population indicating the wildtype flies have again substantial higher fitness than the balancer heterozygotes. For the inbred populations we see now much higher frequencies for the balancers for most populations, particularly for the 3rd chromosome. Qualitatively the picture is similar for both temperatures, although there are somewhat larger fluctuations due to small population size at $29^{\circ} \mathrm{C}$ where almost $40 \%$ of the (replicate) populations went extinct before reaching the 8th generation. If we focus on the TM3 balancer, we see that under temperature stress this balancer reaches appreciable frequencies in seven out of ten populations. To quantify the differences in frequency between outbred MIX and the inbred populations, we calculated the average frequency of the balancer in generation five. For MIX both balancers had disappeared from all ten replicate populations at both 28.5 and $29^{\circ} \mathrm{C}$. For the inbred populations, the averages $( \pm \mathrm{SE})$ over all extant populations at these temperatures for $C y O$ were $0.047 \pm 0.020$ and $0.100 \pm 0.035$ at 28.5 and $29^{\circ} \mathrm{C}$, respectively. For TM3 these values were $0.162 \pm 0.060$ and $0.236 \pm 0.061$, respectively. When calculated for the other generations 
(from generation 3 onwards) the results are qualitatively not different from the fifth generation (data not shown).

For two inbred populations I12 and I15, the TM3 balancer reaches extreme frequencies of nearly $50 \%$. As the balancer can only exist in heterozygotes this means that (nearly) all flies present in the populations are heterozygous and consequently, that at these temperatures homozygous inbred flies for the two populations do not exist. Thus, populations I12 and I15 seem to be fixed for one or a few loci at the 3rd chromosome that cause lethality at high temperature. As this effect is not observed at $25^{\circ} \mathrm{C}$ (see Fig. 3, top), we can only conclude that these two populations are fixed for a conditional lethal (or lethal complex) that is only expressed under high temperature stress.

\section{Rescue experiment}

From the previous experiment it is clear that I12 and I15 show interesting characteristics. Therefore, we used these populations as rescuers for the other eight inbred populations to study the dynamics of genetic rescue and to determine the rescue effect at both 25 and $29^{\circ} \mathrm{C}$. The results are summarized in Fig. 4 and Table 1.

At $25^{\circ} \mathrm{C}$ there is a significant increase in viability by both rescuers (Fig. 4, black bars) in both generation 5 and 10. It increases the viability on average by $7.5-15 \%$. We observe little difference between the two time points (Fig. 4) and our statistical analysis showed there was no effect of generation on the rescue effect. This indicates that the rescue effect is not transient but persists for at least 10 generations. However, there is significant variation between the different inbred populations with respect to the rescue effect. Figure 5 clearly shows this variation for individual rescued populations in generation 5 (the picture for generation 10 is similar, not shown) for both rescuers. First of all, the variation among replicates within populations is generally small

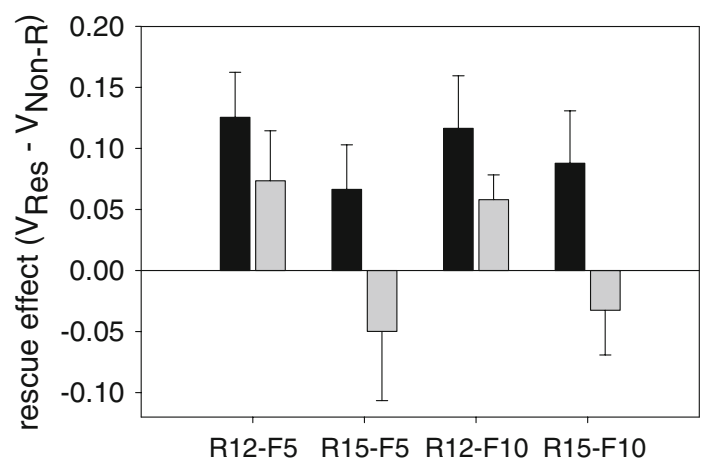

Fig. 4 The average rescue effect $( \pm \mathrm{SE})$ for the two rescuer lines R12 and R15 five generations (F5) and ten generations (F10) after the rescue event. The black bars show the rescue effect at $25^{\circ} \mathrm{C}$ and the grey bars the effect at $29^{\circ} \mathrm{C}$
Table 1 Results of the statistical analysis using mixed linear models as described in "Materials and methods" showing only the results for those variables that significantly affected the rescue effect

\begin{tabular}{lcccl}
\hline Variable & Contrast & $\chi^{2}$-value & df & $P$ \\
\hline Non-rescued viability & 0.611 & 11.47 & 1 & 0.00071 \\
Temperature stress $\left(29^{\circ} \mathrm{C}\right)$ & -0.058 & 32.75 & 1 & $<10^{-7}$ \\
Rescuer R15 & -0.049 & 21.80 & 1 & $<10^{-6}$ \\
Temp. $\times$ rescuer & -0.060 & 4.23 & 1 & 0.0397 \\
\hline
\end{tabular}
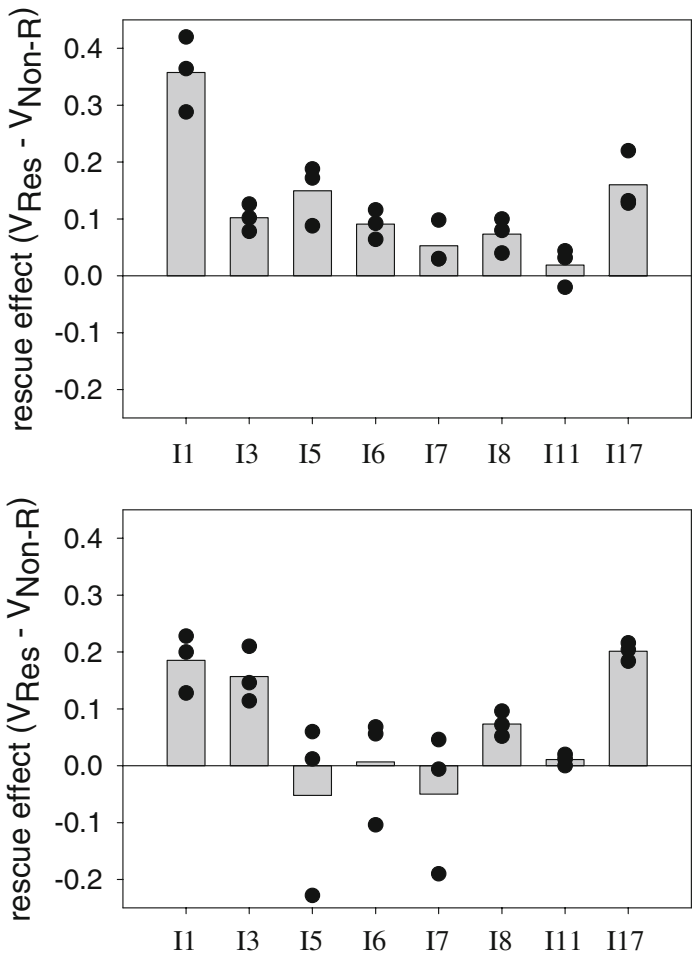

Fig. 5 Variation in the rescue effect among inbred populations five generations after the rescue event for the two rescuers R12 (top) and R15 (bottom). The values of the individual replicates are denoted by filled circles, while the bars show the average over these replicates

compared to the variation among populations. There are a few exceptions for R15 (rescued populations I5, I6 and I7) for which one replicate differs considerably from the other two. The reason for this is unclear at the moment. At the population level we observe large differences between individual inbred populations. Focusing on R12 we see that I1 shows an increase in viability around 30-40\%, whereas I11 shows a non-significant increase of only a few percent. For R15 the picture is similar although the rescue effect is not as large as for R12.

Table 1 shows that the non-rescued viability has a highly significant effect on the increase in viability after the rescued event. This is illustrated in Fig. 6 that shows the relation between non-rescued viability and the rescue effect in both generation five and ten. We observe a strong 

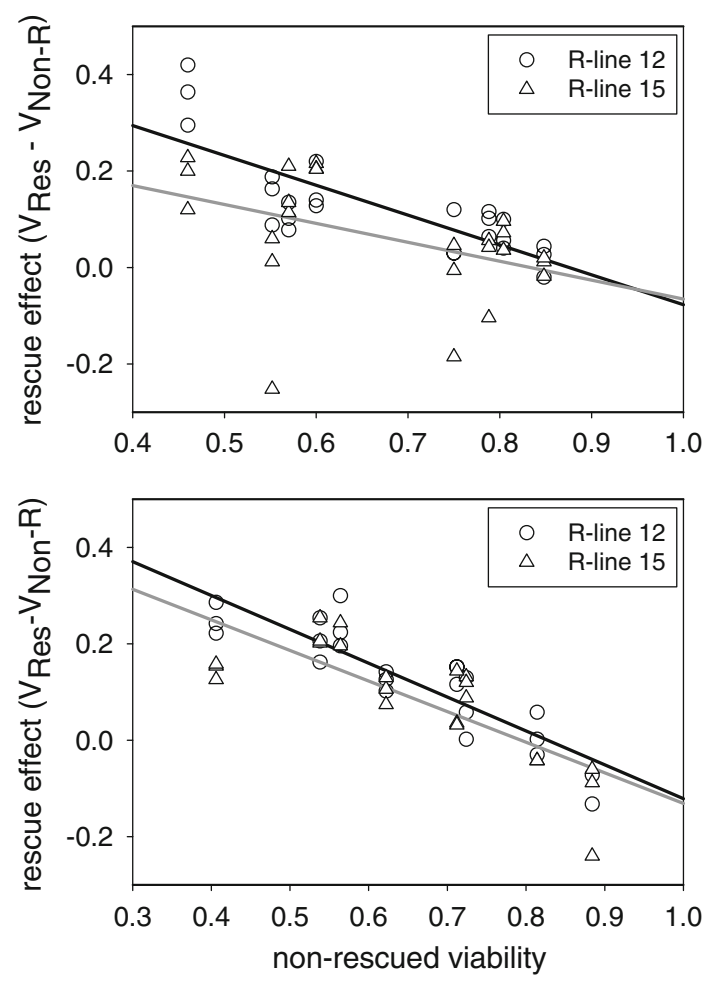

Fig. 6 Relation between the rescue effect and non-rescued viability for the two rescuer lines in generation F5 (top) and F10 (bottom) and the linear regression through these for R12 (black) and R15 (grey)

negative relationship showing that rescuing is more successful when the non-rescued viability is lower.

As R12 and R15 both carry a conditionally expressed recessive lethal that is mildly deleterious at $25^{\circ} \mathrm{C}$ and lethal at $29^{\circ} \mathrm{C}$, introgression of this lethal at $25^{\circ} \mathrm{C}$ (the temperature the rescue experiment was done) will affect the rescue effect at $29^{\circ} \mathrm{C}$. Figure 4 shows that indeed the rescue effect decreases when the rescued populations are tested at this high temperature (grey bars). Table 1 shows that this effect is highly significant. To show that the rescued populations have become more sensitive to high temperature stress we calculated the cost of stress being the difference in viability between 25 and $29^{\circ} \mathrm{C}$ averaged over all populations. In generation 5 means $( \pm \mathrm{SE})$ were $0.151 \pm 0.023,0.203 \pm 0.025$ and $0.280 \pm 0.048$ for nonrescued, rescued by R12 and rescued by R 15 , respectively. In generation 10 the data were $0.114 \pm 0.033,0.160 \pm$ 0.024 and $0.234 \pm 0.027$ for non-rescued, rescued by R12 and rescued by $\mathrm{R} 15$, respectively. In both generations the cost of stress is indeed the lowest for the non-rescued populations and the highest for populations rescued by R15. A one-way analysis of variance revealed that the differences are significant both in generation $5\left(F_{2,23}=\right.$ 3.68, $P=0.04)$ and generation $10 \quad\left(F_{2,23}=4.74\right.$, $P=0.02$ ). A multiple comparison of means showed that the non-rescued populations differed significantly from those rescued by R15, while R12 did not differ significantly from both of these in both generations.

Figure 4 also indicates a difference between the rescuers: R15 shows on average a smaller rescue effect than R12. In agreement, our statistical analysis shows a significant effect of the rescuer with respect to the rescue effect as well as a significant interaction between rescuer and temperature (Table 1). Basically, R15 seems to be less effective at $25^{\circ} \mathrm{C}$ (Fig. 4, black bars) but at the same time populations rescued by R15 are more strongly affected by the stress temperature (Fig. 4, grey bars). We expected that the rate of introgression of the lethal might be positively correlated to the size of the rescue effect. However, the correlation between these two factors turned out to be not significant at both generation 5 and 10 (data not shown).

\section{Recurrent inbreeding and pupal mortality}

The increased cost of stress after the rescue event is most probably due to introgression of the conditional lethal into the rescued populations at the benign temperature. To investigate the fate and consequence of such (a) gene(s) under renewed inbreeding, we tested the difference in preadult mortality of inbred lines from both the rescued and non-rescued populations. As it was at that time clear that the conditional lethal caused specifically mortality in the pupal stage at $29^{\circ} \mathrm{C}$ only (and not at $25^{\circ} \mathrm{C}$ ), we used pupal mortality as measure instead of pre-adult mortality. As a broad picture, Fig. 7 a shows the increase in pupal mortality at $29^{\circ} \mathrm{C}$ compared to $25^{\circ} \mathrm{C}$ averaged over 7 inbred populations (no inbred lines from population I5 did survive the 6 generations of recurrent full-sib mating). This is shown for the different types of populations in the experiment (see Fig. 2): the original populations non-rescued populations (N-R;NI), the non-rescued populations after six additional rounds of full-sib mating (N-R;I), and the rescued populations after additional inbreeding (R12;I and R15;I). As the experimental design was quite unbalanced (different degrees of replication at different levels), we analysed the data for the different factors separately with mixed linear models. First we tested for the effect of the additional inbreeding on pupal mortality and found a significant effect $\left(\chi^{2}=4.49, \mathrm{df}=1\right.$, $P=0.034)$. Comparing N-R;N-I with N-R;I shows that the 6 generations of additional inbreeding more than doubles the level of pupal mortality (Fig. 7a). This indicates that inbreeding per se increases pupal mortality at temperature stress. Second, the comparison between additionally inbred non-rescued populations (N-R;I) and additionally inbred rescued populations (R12;I and R15;I) showed that rescuing did significantly increase the pupal mortality at $29^{\circ} \mathrm{C}$ $\left(\chi^{2}=22.52, \mathrm{df}=1, P<0.0001\right)$. As the inbreeding level is the same for all populations this indicates that the increase is due to the introgression of the conditional lethal 

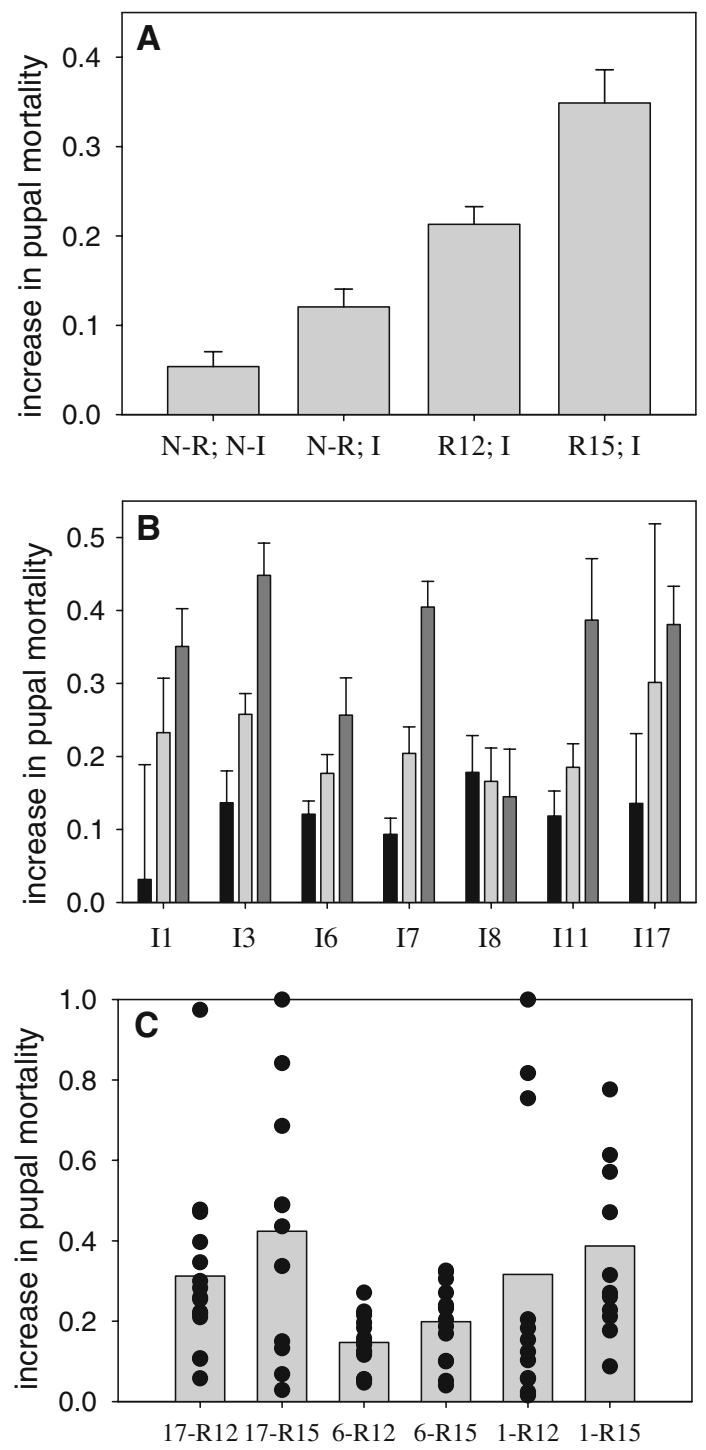

Fig. 7 Average increase in pupal mortality at $29^{\circ} \mathrm{C}$ compared to at $25^{\circ} \mathrm{C}$ at three levels. a Shows the mean $( \pm \mathrm{SE})$ averaged over all inbred populations for four different groups: N-R; N-I: original inbred populations, N-R; I: non-rescued populations but additionally inbred, R12; I: rescued by R12 and additionally inbred and R15; I: rescued by R15 and additionally inbred. b Shows the average $( \pm$ SE) for the different populations tested for N-R; I (black bars), R12; I (light grey bars) and R15; I (dark grey bars). c shows the variation in the increase of pupal mortality among individual inbred lines for three inbred populations (I17, I6 and I1) rescued by either R12 or R15. The grey bars denote the average over the replicate inbred lines (filled black circles)

into the rescued populations. Third, we tested the differences due to the rescuers (R12 vs. R15) and found this difference to be highly significant $\left(\chi^{2}=21.58\right.$, $\mathrm{df}=1$, $P<0.0001)$. This could mean that the introgression of the conditional lethal was more successful for R15 than for $\mathrm{R} 12$. This is not in line with our previous finding that $\mathrm{R} 12$ is a better rescuer than R15, but would explain why the rescue effect of R15 decreases much more under temperature stress compared to R12 (Fig. 4).

At the level of the individual populations, we observed significant variation both among and within populations (Fig. $7 b-c$ ). Figure $7 b$ shows the variation in the increase in pupal mortality among lines for the non-rescued compared to the rescued populations. This figure again illustrates clearly the difference between the rescued and nonrescued populations and also that the mortality in general is higher for R15 than for R12. The exception is population I8 that shows a relatively high level of pupal mortality as nonrescued population, but does not show any increase in mortality after the rescue event.

The within population variation is shown for a few selected cases in Fig. 7c. It is clear that for I17 and I1 the individual inbred lines made from these populations ten generations after the rescue event show a highly variable effect on pupal mortality. For some inbred lines pupal mortality does not increase at $29^{\circ} \mathrm{C}$ compared to $25^{\circ} \mathrm{C}$, while for others it increases to $100 \%$ pupal mortality. In fact, for these two populations we see a bi-model or near uniform distribution of the increase in pupal mortality due to high temperature. This is compatible with the idea that the conditional lethal has introgressed to a reasonable high frequency in these populations and, as we did the inbreeding at $25^{\circ} \mathrm{C}$, has become subject to severe genetic drift during the six generations of full-sib matings leading to extreme frequencies in individual inbred lines. A contrasting picture is observed for population I6 for which the variation among inbred lines is relatively small, suggesting that in this case the conditional lethal did not introgress into the population, or reached only very low frequencies, so that the applied genetic drift did not cause variation among lines. This seems to agree with our observation concerning the size of the rescue effect for this population (Fig. 5).

\section{Discussion}

Introgression of deleterious alleles

Under benign conditions we observed that in eight out of ten populations the balancers disappeared from the rescued populations although at a slower rate than in the outbred MIX populations (Fig. 3). This means that inbred wildtype homozygotes have a lower fitness than wildtype flies of the outbred population, but the fitness loss is not such that that it leads to a stable polymorphism in the populations and in the end the balancers are lost. This does not necessarily imply that there is no inbreeding depression in these populations because the inbred homozygotes have a similar or larger fitness than the balancer heterozygotes. It is well 
known that balancer heterozygotes have a significantly lower fitness than wildtype heterozygotes (Tracey and Ayala 1974; Mackay 1985). This reduction in fitness has been estimated to be around $20 \%$. This means that even if the inbreeding depression is not much less than $20 \%$ per chromosome, the balancer will still disappear from the inbred population.

At the same time we observed that under thermal stress the balancers reached much higher frequencies and persisted at appreciable frequencies in most of the rescued populations, particularly so for the $T M_{3}$ balancer (Fig. 3). Consequently, these populations suffer considerably more from inbreeding depression under stress than under benign conditions. This can only be explained assuming that, compared to $25^{\circ} \mathrm{C}$, under stress the fitness of the inbred wildtypes decreases relative to that of the balancer heterozygotes. In other words, inbreeding depression increases under the applied temperature stress in most inbred populations. This agrees well with previous findings for this population (Bijlsma et al. 1999) and adds to an increasing number of data showing that the negative effects of inbreeding greatly increase under stress conditions (Dahlgaard and Hoffmann 2000; Keller et al. 2002; Ambruster and Reed 2005).

The balancer experiment showed that the heterosis accompanying genetic rescue can be a powerful force to allow even highly deleterious alleles, in this case a recessive lethal, to increase in frequency in the rescued populations. This force is particularly strong when the fitness of the rescued population is low, as I1 and I5 were among the populations showing the highest inbreeding depression for viability. Most extreme was the situation for inbred populations I12 and I15 at $29^{\circ} \mathrm{C}$ where only balancer heterozygotes were viable under these conditions and the recessive lethal carried by the immigrants did reach a frequency of 0.50 in the rescued populations. Thus when deleterious alleles from the immigrants are tightly linked to the genes that are responsible for the heterosis and mask inbreeding depression, this deleterious gene can hitchhike along and reach appreciable frequencies in the recipient population.

For the increase in frequency of the deleterious alleles linkage to the genes that are responsible for the heterosis is essential. In that light, our balancer experiment is somewhat artificial as both the entire 2 nd and 3 rd chromosome each form one linkage group. Consequently, the heterotic force of all loci at a balancer chromosome that contribute to heterosis are combined in their effort and inseparable. For inversion free chromosomes, recombination would uncouple these loci in later generations whereby the heterotic force becomes smaller as individuals will be heterozygous for only a subset of the loci that are responsible for the heterosis in the first generation hybrids. This explains why the rescue effect is generally expected to be the strongest in the first generation(s) after a rescue event but is diluted in subsequent generations (Lynch 1991). Because of this, there is little chance that recessive, highly deleterious alleles from the immigrant genome would reach appreciable frequencies in rescued population like observed in our balancer experiment, unless it is tightly linked to a locus with a very strong heterotic effect. However, mildly deleterious alleles, probably responsible for the greater part of the inbreeding depression observed in inbred populations (Hedrick 1994), still are expected to be able to increase substantially in frequency when linked to a locus causing the heterotic effect.

That hitchhiking can be responsible for an increase of alleles from the immigrant into the rescued population is clearly shown in our pupal mortality experiment. As mortality during the pupal stage is the specific phenotype of lethal homozygotes, we can assume that the increase in pupal mortality at $29^{\circ} \mathrm{C}$ in the rescued populations compared to the non-rescued populations (Fig. 7b) is mainly due to the increased frequency of the conditional lethal that was present in the rescuers R12 and R15. From this, we infer that this lethal must have reached substantial frequencies in most rescued populations. Also the data on the increase of the cost of stress support this finding. However, under the temperature conditions the rescued populations were maintained $\left(25^{\circ} \mathrm{C}\right)$, the lethal effect is not expressed and the lethal allele is most probably mildly deleterious. So, an increase in frequency of the conditional lethals can only be explained by hitchhiking along with the loci that are under selection and responsible for the heterotic effect. This is in agreement with the finding that rescue events often show a strong increase in the heterozygosity for neutral microsatellites (Madsen et al. 1999; Bouzat et al. 2009).

In conclusion, there is little doubt that genetic rescue of greatly inbred populations might cause a disproportional increase in the frequency of mildly deleterious alleles that are carried by the immigrants.

Can we prevent this to happen by carefully selecting the most fit individuals of a donor population as immigrants? The answer will be no. Assuming that inbreeding depression for the greater part is due to homozygosity for recessive deleterious alleles, species that suffer from high inbreeding depression when confined to small population size necessarily are species that carry a high amount of lethal equivalents. Half of these lethal equivalents are thought to be caused by many loci that have small fitness effects (Charlesworth and Charlesworth 1999; Lynch et al. 1999). As a consequence, all individuals of those species that display high levels of inbreeding depression upon inbreeding are expected to carry part of this load in heterozygous condition and, as such, escape the scrutiny of selection. 
The rescue effect

Our data show that genetic rescue can be effective to increase the fitness of inbred populations, particularly when the non-rescued fitness was low (Fig. 6). This is, of course, not unexpected as it is impossible to improve the viability of populations that have already a viability near the maximum. The viability assay used here, normally reveals a viability between 0.75 and 0.90 for fully outbred populations. This implies that the inbred populations that showed a viability above 0.75 when non-rescued will show very little gain from genetic rescue. In fact, if we add the rescue effect to the non-rescued viability (see Fig. 6) we find that most rescued populations have attained a viability of 0.75 or more after the rescue event. This means that they all reached a viability (nearly) similar to that of outbred populations. This suggests that the observed negative correlation between the non-rescued viability and the rescue effect is for the greater part due to the methodology.

As we were interested in the more long-term consequences of genetic rescue, we determined the rescue effect 5 and 10 generations after the rescue event and observed that the increase in fitness persisted during this interval. As such, we do not know the dynamics during the first generation directly after the rescue event. It could be that the fitness increase due to immigration was larger during the first generations and then declined to the value we observed at generation 5. This would agree with the idea that the heterosis effect becomes diluted in later generations (Lynch 1991; Hooftman et al. 2007). On the other hand, if the population size of the recipient population is not too small, the number of heterozygotes may be expected to increase over the first consecutive generations and the fitness could have steadily climbed to the value observed in generation 5. Whatever, our data show that the rescue effect is not a short-lived and transient phenomenon but persists for longer periods, at least when population sizes are kept reasonably high after the rescue event.

A difference between our approach and what will be normal practice in managing endangered populations is that we used individuals from another highly inbred population as immigrants. To what extent this did affect the outcome of the experiment is not clear at the moment. However, whereas Ball et al. (2000) showed that outbred individuals performed significantly better than inbred immigrants, Saccheri and Brakefield (2002) showed that even highly inbred immigrants performed quite well as rescuer. The outcome may also depend on how the rescue was done. Ball et al. (2000) used a single male of immigrant that had to compete with other males for access to the females and found that the vigour of the immigrant male was decisive for the outcome. Saccheri and Brakefield (2002) started with one resident female that was mated to an immigrant male and showed that heterosis was the driving force. Our method, introducing virgin females into the resident populations resembles the latter experiment and we conclude that heterosis is also the driving force in our case. Based on the findings that the rescue effect decreased significantly when tested at $29^{\circ} \mathrm{C}$ (Fig. 4) and the observed high pupal mortality at the same temperature (Fig. 7), we infer that the conditional lethal, and by extrapolation also other parts of the immigrant genome, had attained appreciable frequencies in most of the rescued populations. This agrees well with the results of Saccheri and Brakefield (2002).

The rescue effect also depended significantly on the rescuer used. R12 increased the fitness of the rescued populations to a higher level than R15. On the other hand, the conditional lethal seems to have introgressed to a significant higher frequency in the rescued population when $\mathrm{R} 15$ was used than using R12. This can be inferred from the fact that the inbred populations rescued by R 15 showed a larger decrease in the rescue effect (Fig. 4) and a higher increase in pupal mortality (Fig. 7) when tested at $29^{\circ} \mathrm{C}$. It is conceivable that the two rescuer populations differed considerably in genetic make up (it is at the moment even unknown if the conditional lethals are the same). Another important factor in this respect is that we focused on a single fitness component, pre-adult viability. However, many other fitness components in Drosophila show high levels of inbreeding depression upon inbreeding (Lynch and Walsh 1998; Kristensen and Sorensen 2005; Kristensen et al. 2010). It might well be that some of these, like fecundity or sterility, also showed strong heterosis upon genetic rescue and affected the introgression of the immigrant genome. To evaluate the success of genetic rescue more fully it is necessary to study many more fitness components.

Notwithstanding, these uncertainties, our results show that genetic rescue is successful, like found in other cases, and that the positive effect on fitness is not transient but persists over longer periods. This agrees well with the findings of Willi et al. (2007).

\section{Recurrent inbreeding}

Genetic rescue is applied as a management measure to overcome the decrease in fitness of small isolated populations due to genetic drift and inbreeding. However, the long-term benefits of this approach are only warranted if it is accompanied by a substantial increase in population size. If the population stays relatively small and genetic drift and inbreeding are still significant forces, the fitness is expected to decrease again in subsequent generations (Liberg et al. 2005; Robert et al. 2007). Even worse, as mildly deleterious alleles present in the immigrants might have reached 
appreciable frequencies in the rescued populations, renewed inbreeding and genetic drift may lead to even more severe inbreeding depression. This is due to the fact that, assuming the deleterious alleles present behave near neutral under genetic drift $(N \mathrm{~s}<1)$, the fixation probability is directly related to the frequency the deleterious alleles had attained in the rescued population: the probability of fixation is the same as the initial allele frequency (Kimura 1983). Even in a situation where natural selection is partly effective, still a higher frequency of the deleterious alleles would mean a higher probability of fixation.

We have mimicked this process by subjecting the rescued populations to renewed inbreeding. The conditional lethal(s) from both rescuers will have reached substantial frequencies while hitchhiking along with the genes responsible for the heterosis (see Fig. 7). Because the conditional lethals are mildly deleterious or nearly neutral at $25^{\circ} \mathrm{C}$, the temperature at which the inbreeding was done, the deleterious alleles will be subject to genetic drift and can reach high frequencies in individual inbred lines or even go to fixation (Fig. 7c). When such lines are subsequently tested at $29^{\circ} \mathrm{C}$ they show low survival and readily will go extinct.

The same process is expected to affect other mildly deleterious alleles that were present in the rescuers. Thus, initially successful genetic rescue followed by subsequent inbreeding and genetic drift in many cases is expected to lead to even more severe loss of fitness in the rescued population than it originally experienced before the rescue event.

\section{Inferences for conservation management}

In this paper we evaluated the dynamics of genetic rescue and show that the positive aspect of genetic rescue is a long-term increase in fitness of the rescued population. However, this will be accompanied by an increase in the frequency of recessive deleterious alleles carried by the immigrants, which, as the population experiences recurrent inbreeding and drift again, may endanger persistence of the rescued population even more. However, this will only be a problem when the deleterious alleles present in the rescuers reach reasonable high frequencies. This can be overcome by using a sufficient number of unrelated immigrants and ensure that they all contribute more or less equally to the next generations. As there are many loci in large populations that carry deleterious alleles but these generally have low frequencies, the immigrants will all carry deleterious alleles but mostly different ones. Although these still may increase in frequency due to the force of heterosis, the increase will be much lower for individual alleles. If all immigrants contribute equally to the next generations, the increase of an allele present in one out of ten immigrants is expected to reach an average frequency of only $10 \%$ of that when this individual was used as a single immigrant. Although introducing "many" immigrants may threaten the genetic integrity of the recipient population somewhat, it will also prevent a reduction of $N_{e}$ (see Hedrick and Fredrickson 2010) as none of the immigrant genomes will become predominant in the rescued population. If for other reasons the use of few immigrants is preferable, it is recommended to repeat the immigration of unrelated immigrants in the subsequent generations to prevent certain immigrant genomes, and thereby also its recessive mildly deleterious alleles, to become overrepresented.

A second issue is that genetic rescue necessarily has to be accompanied by demographic and environmental measures (see Robert et al. 2007). If population size is only temporarily boosted and genetic drift and inbreeding take precedence again in later generations, the situation rapidly can become worse than before the rescue event. This means that transplantation of individuals to endangered populations only makes sense when habitat alterations and increasing connectivity warrant that the population size is permanently boosted. If not, genetic rescue has to be regularly repeated.

Although there are quite a number of risks connected with genetic rescue (Tallmon et al. 2004; Edmands 2007; Hedrick and Fredrickson 2010) including the ones discussed here, genetic rescue has proven to be a successful method to increase the fitness of genetically eroded populations and to improve their persistence (Hedrick 1995; Westemeier et al. 1998; Madsen et al. 1999; Willi et al. 2007; Bouzat et al. 2009; Hedrick and Fredrickson 2010). Thus if populations decline in numbers partly due to genetic problems and because of this have a high probability of going extinct in the near future, genetic rescue offers a viable management option, notwithstanding the risks connected to it.

Acknowledgments We thank Phil Hedrick, Torsten Kristensen and an anonymous reviewer for valuable suggestions to improve earlier versions of the manuscript. We thank Anneke Boerema, Hervé Rohier and Rogier Houwerzijl for technical assistance. Part of the work was done while RB was visiting the stress group of Volker Loeschcke that was made possible by a grant from the University of Aarhus (DK). This work has greatly profited from discussions within the framework the Research Networking Programme ConGen funded by the European Science Foundation.

Open Access This article is distributed under the terms of the Creative Commons Attribution Noncommercial License which permits any noncommercial use, distribution, and reproduction in any medium, provided the original author(s) and source are credited. 


\section{References}

Ambruster P, Reed DH (2005) Inbreeding depression in benign and stressful environments. Heredity 95:235-242

Bakker J, Van Rijswijk MEC, Weissing FJ, Bijlsma R (2010) Consequences of fragmentation for the ability to adapt to novel environments in experimental Drosophila metapopulations. Conserv Genet (this issue). doi:10.1007/s10592-010-0052-5

Ball SJ, Adams M, Possingham HP, Keller MA (2000) The genetic contribution of a single male immigrant to small, inbred populations: a laboratory study using Drosophila melanogaster. Heredity 84:668-677

Bijlsma R, Bundgaard J, Van Putten WF (1999) Environmental dependence of inbreeding depression and purging in Drosophila melanogaster. J Evol Biol 12:1125-1137

Bijlsma R, Bundgaard J, Boerema A (2000) Does inbreeding affect the extinction risk of small populations? Predictions from Drosophila. J Evol Biol 13:502-514

Bouzat JL, Johnson JA, Toepfer JE, Simpson SA, Esker TL, Westemeier RL (2009) Beyond the beneficial effects of translocations as an effective tool for the genetic restoration of isolated populations. Conserv Genet 10:191-201

Caughley G (1994) Directions in conservation biology. J Anim Ecol 63:215-244

Ceballos G, Ehrlich PR (2002) Mammal population losses and the extinction crisis. Science 296:904-907

Charlesworth D, Charlesworth B (1999) The genetic basis of inbreeding depression. Genet Res 74:329-340

Crawley MJ (2007) The R Book. Wiley, Chichester

Dahlgaard J, Hoffmann AA (2000) Stress resistance and environmental dependency of inbreeding depression in Drosophila melanogaster. Conserv Biol 14:1187-1192

Dobzhansky T, Pavlovsky O (1958) Interracial hybridization and breakdown of coadapted gene complexes in Drosophila paulistorum and Drosophila willistoni. Proc Natl Acad Sci USA 44: 622-629

Dobzhansky T, Pavlovsky O, Spassky B, Spassky N (1955) Genetics of natural populations. XXIII. Biological role of deleterious recessives in populations of Drosophila. Genetics 40:781-796

Ebert D, Haag C, Kirkpatrick M, Riek M, Hottinger JW, Pajunen VI (2002) A selective advantage to immigrant genes in a Daphnia metapopulation. Science 295:485-488

Edmands S (2007) Between a rock and a hard place: evaluating the relative risks of inbreeding and outbreeding for conservation and management. Mol Ecol 16:463-475

Falconer DS, Mackay TCF (1996) Introduction to quantitative genetics, 4th edn. Pearson Education Ltd, Harlow, England

Frankham R (1995) Conservation genetics. Ann Rev Genet 29:305-327

Hedrick PW (1994) Purging inbreeding depression and the probability of extinction: fullsib mating. Heredity 73:363-372

Hedrick PW (1995) Gene flow and genetic restoration: the Florida panther as a case study. Conserv Biol 9:996-1007

Hedrick PW (2005) "Genetic restoration": a more comprehensive perspective than "genetic rescue". Trends Ecol Evol 20:109

Hedrick PW, Fredrickson RJ (2010) Genetic rescue guidelines with examples from Mexican wolves and Florida panthers. Conserv Genet (this issue). doi:10.1007/s10592-009-9999-5

Hedrick PW, Kalinowski S (2000) Inbreeding depression and conservation biology. Ann Rev Ecol Syst 31:139-162

Hedrick PW, Lacy RC, Allendorf FW, Soulé ME (1996) Directions in conservation biology: comments on Caughley. Conserv Biol 10: $1312-1320$

Hooftman DAP, Jong MJD, Oostermeijer JGB, Den Nijs HJCM (2007) Modeling the long-term consequences of crop-wild relative hybridization: a case study using four generations of hybrids. J Appl Ecol 44:1035-1045

Keller LF, Waller DM (2002) Inbreeding effects in wild populations. Trends Ecol Evol 17:230-241

Keller LF, Grant PR, Grant BR, Petren K (2002) Environmental conditions affect the magnitude of inbreeding depression in survival of Darwin's finches. Evolution 56:1229-1239

Kimura M (1983) The neutral theory of molecular evolution. Cambridge University Pres, Cambridge

Kristensen TN, Sorensen AC (2005) Inbreeding-lessons from animal breeding, evolutionary biology and conservation genetics. Anim Sci 80:121-133

Kristensen TN, Pedersen KS, Vermeulen CJ, Loeschcke V (2010) Research on inbreeding in the 'omics' era. Trends Ecol Evol 25: 44-52

Leducq JB, Gosset CC, Poiret M, Hendoux F, Vekemans X, Billiard S (2010) An experimental study of the $S$-Allele effect in the self incompatible Biscutelle neustriaca (Brasicaceae). Conserv Genet (this issue). doi:10.1007/s10592-010-0055-2

Liberg O, Andrén H, Pederson H-C, Sand H, Sejbeg D, Wabakken P, Åkesson M, Bensch S (2005) Severe inbreeding depression in a wild wolf (Canis lupus) population. Biol Lett 1:17-20

Lindsley DL, Zimm GG (1992) The genome of Drosophila melanogaster. Academic Press, San Diego

Lynch M (1991) The genetic interpretation of inbreeding depression and outbreeding depression. Evolution 45:622-629

Lynch M, Walsh B (1998) Genetics and analysis of quantitative traits. Sinauer Associates, Sunderland

Lynch M, Blanchard J, Houle D, Kibota T, Schultz S, Vassilieva L, Willis J (1999) Perspective: spontaneous deleterious mutations. Evolution 53:645-663

Mackay TFC (1985) A quantitative genetic analysis of fitness and its components in Drosophila melanogaster. Genet Res Camb 47: 59-70

Madsen T, Shine R, Olsson M, Wittzell H (1999) Restoration of an inbred adder population. Nature 402:34-35

Maynard Smith J, Haigh J (1974) The hitchhiking effect of a favourable gene. Genet Res 23:23-35

Newman D, Pilson D (1997) Increased probability of extinction due to decreased genetic effective population size: experimental populations of Clarkia pulchella. Evolution 51:354-362

R Development Core Team (2005) R: a language and environment for statistical computing. R Foundation for Statistical Computing, Vienna, Austria

Robert A, Couvet D, Sarrazin F (2007) Integration of demography and genetics in population restoration. Ecoscience 14: $463-471$

Rohmer C, David JR, Moreteau B, Joly D (2004) Heat induced male sterility in Drosophila melanogaster: adaptive genetic variations among geographic populations and role of the $\mathrm{Y}$ chromosome. J Exp Biol 207:2735-2743

Saccheri IJ, Brakefield PM (2002) Rapid spread of immigrant genomes into inbred populations. Proc R Soc B 269:10731078

Saccheri I, Kuussaari M, Kankare M, Vikman P, Fortelius W, Hanski I (1998) Inbreeeding and extinction in a butterfly metapopulation. Nature 392:491-494

Spielman D, Brook BW, Frankham R (2004) Most species are not driven to extinction before genetic factors impact them. Proc Natl Acad Sci USA 101:15261-15264

Sved JA, Ayala FJ (1970) A population cage test for heterosis in Drosophila melanogaster. Genetics 66:97-113

Tallmon DA, Luikart G, Waples R (2004) The alluring simplicity and complex reality of genetic rescue. Trends Ecol Evol 19:489-496 
Tobari I (1966) Effects of temperature on the viabilities of homozygotes and heterozygotes for second chromosomes in Drosophila melanogaster. Genetics 54:783-791

Tracey ML, Ayala FJ (1974) Genetic load in natural populations: is it compatible with the hypothesis that many polymorphisms are maintained by natural selection? Genetics 77:569-598

Vermeulen CJ, Bijlsma R (2004a) Changes in mortality patterns and temperature dependence of lifespan in Drosophila melanogaster caused by inbreeding. Heredity 92:275-281

Vermeulen CJ, Bijlsma R (2004b) Characterization of conditionally expressed mutants affecting age-specific Drosophila melanogaster: lethal conditions and temperature-sensitive periods. Genetics 167:1241-1248

Vermeulen CJ, Bijlsma R (2006) Changes in genetic architecture during relaxation in Drosophila melanogaster selected on divergent virgin life span. J Evol Biol 19:216-227

Vilà C, Sundqvist A-K, Flagstad $\varnothing$, Seddon J, Björnerfeldt S, Kojola I, Casulli A, Sand H, Wabakken P, Ellegren H (2003) Rescue of a severely bottlenecked wolf (Canis lupus) population by a single immigrant. Proc R Soc B 270:91-97

Wang JL (2000) Effects of population structures and selection strategies on the purging of inbreeding depression due to deleterious mutations. Genet Res Camb 76:75-86

Wang J, Hill WG, Charlesworth D, Charlesworth B (1999) Dynamics of inbreeding depression due to deleterious mutations in small populations: mutation parameters and inbreeding rate. Genet Res 74:165-178

Westemeier RL, Brawn LD, Simpson SA, Esker TL, Jansen RW, Walk JW, Kershner EL, Bouzat JL, Paige KN (1998) Tracking the long-term decline and recovery of an isolated population. Science 282:1695-1698

Willi Y, van Kleunen M, Dietrich S, Fischer M (2007) Genetic rescue persists beyond the first-generation outbreeding in small populations of a rare plant. Proc R Soc B 274:2357-2364 